\title{
Methods of preserving platelets: cryopreservation as a reasonable option for its implementation in Argentina
}

\author{
Jhon Alexander Avila Rueda ${ }^{1 *}$, Pablo Young ${ }^{2}$, Cristina Duboscq ${ }^{1}$ \\ ${ }^{1}$ Hematology Department, Buenos Aires British Hospital, Buenos Aires, Argentina \\ ${ }^{2}$ Internal Medicine Department, Buenos Aires British Hospital, Buenos Aires, Argentina
}

\section{Abstract}

Platelets are one of the most widely used blood components in transfusion medicine, given their importance in the prevention of bleeding disorders in patients with multifactorial thrombocytopenia and Asian non-immunological etiology, as well as in the management of traumatic or blood-related surgical treatments. Given its extensive use and the constant demand for it to be used within the different procedures associated with medicine, it is the objective of both hemotherapy services and blood banks to ensure that blood product components are constantly available to meet such requirements.

The main challenge with this resource is its short useful life (5-7 days) which limits its availability, especially in the Latin American context where the production of blood components as well as the resources allocated for this purpose by state entities to blood banks, are generally limited. Cryopreservation at $-80^{\circ} \mathrm{C}$ using dimethylsulfoxide as a preservative solution is a technique used since the 1950 s to prolong platelet survival, the improvement of which has been remarkable in recent years, thereby constituting an alternative solution to this problem.

Key words: platelets, hemotherapy, cryopreservation, transfusion medicine

Acta Haematologica Polonica 2021; 52, 3: 163-168

\section{Introduction}

Platelets that will be used in transfusion procedures can be extracted from the centrifugation of a unit of whole blood to initially obtain platelet-rich plasma which will be centrifuged again until obtaining a platelet product that will later be stored under the conditions that we specify below. Another way to obtain it is through a procedure called apheresis where after processing 4-5 liters of whole blood, a platelet concentrate is obtained, with an expected minimum count of $300 \times 10^{9} / \mathrm{L}[1]$.

Thus, in transfusion medicine, the use of platelets can be classified according to two main objectives: prophylaxis for the prevention of bleeding in patients with thrombocytopenia or platelet dysfunction, and therapeutics for the management of major bleeding in conjunction with other blood components, as well as support for thrombocytopenia of non-immunological variable causes.

On the other hand, the criteria that define an indication for platelet transfusion are: people with platelet count less than $7,000 / \mu \mathrm{L}$ with no bleeding, people with platelet count less than $20,000 / \mu \mathrm{L}$ (with evidence of bleeding, fever, sepsis, or sustained high blood pressure), people with platelet count less than $50,000 / \mu \mathrm{L}$ prior to major surgery, invasive procedure or in acute active leukemic phase (promyelocytic leukemia associated with coagulopathy), and people with platelet count less than $100,000 / \mu \mathrm{L}$ prior to neurosurgery or with evidence of central nervous system bleeding [1, 2].

The great limitation of platelets conserved in the conventional way is the difficulty in maintaining the viability of

\footnotetext{
*Address for correspondence: Jhon Alexander Avila Rueda, Hematology Department, Buenos Aires British Hospital, Perdriel 74 Autonomous City of Buenos Aires, Argentina, e-mail: jaavilar@gmail.com
} 
this blood component for long periods of time, which has motivated the search for new strategies to prolong its survival and functionality.

The objective of this work was to review the current conditions for the conservation of platelets, including the conventional form and also other strategies such as cryopreservation, describing its probable benefits and clinical utility.

\section{Platelet characteristics}

Platelets, also known as thrombocytes, were recognized for the first time by Friederich Arnold in 1845 from observation through a low-resolution microscope. Then between 1846 and 1873 and based on descriptions provided by Zimmerman, Shultze, Osler and Vulpian, platelets became known for their ability to adhere and clump together to form platelet aggregates [3, 4]. From the histological point of view, they are cytoplasmic fractions originating from the fragmentation of their immediate precursor, the megakaryocyte through an endomytotic process. Such fragments measure 1-3 $\mu \mathrm{m}$ in diameter and lack a nucleus, although they contain organelles such as lysosomes, mitochondria and peroxisomes, as well as alpha granules and dense granules [5]. It also contains membrane receptors whose function and responses are determined by the ligand for which this was designed, for example, the P2Y receptors for adenosine diphosphate, glycoprotein (GP) Ib/IX/V for von Willebrand factor (VWF), PAR1 and 2 for thrombin and GP IIb/Illa for fibrinogen, among others (Figure 1).

It participates in the hemostasis process by interacting on the area of vascular endothelial damage through adherence and subsequent platelet activation and aggregation, characteristic steps inherent to primary hemostasis. Of course, platelets are not limited to this single step, because both their membranes and the content of their granules are also the basis of a series of more complex processes, such as the activation of the coagulation cascade $[6,7]$.

As for the platelet activation process, this begins from the moment when the integrity of the endothelium is lost, at which time there is exposure of collagen fibers which interact with the VWF (particularly types I, III and VI collagen) and other proteins providing an optimal surface for platelet adhesion by binding through its GP Ib receptor [8]. Subsequently, a signaling process occurs as well as the acquisition of a flat shape by the platelet, product of a rearrangement of the actin cytoskeleton while the activation of phospholipase A2 occurs, releasing arachidonic acid which is an essential component for the production of thromboxane A2. This, together with other substances such as adenosine diphosphate (ADP) and serotonin in addition to activation of receptor ligand binding sites GP Ilb/IIla, favors the process of amplification of activation and aggregation by interacting with receptors on the membrane surface of other platelets, increasing it (Figure 2).

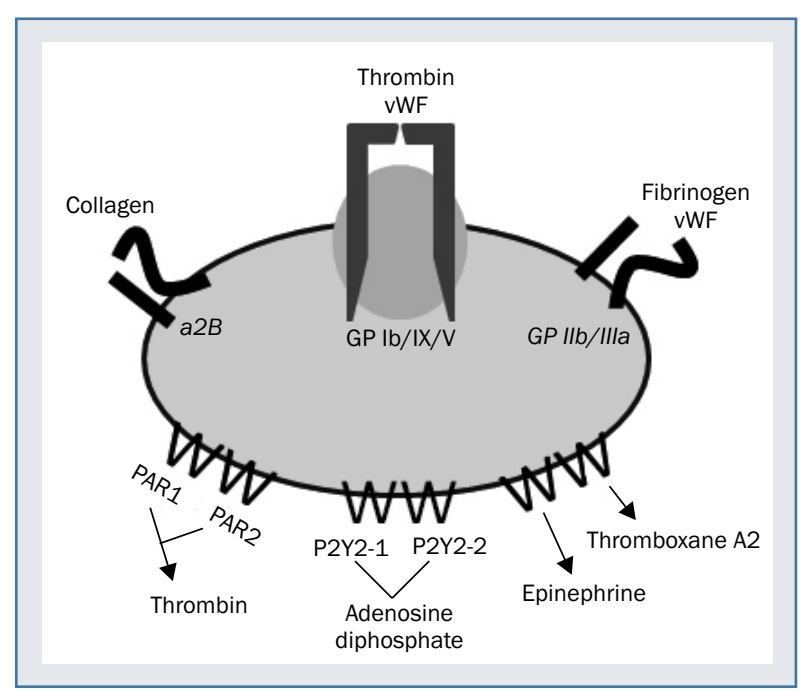

Figure 1. A platelet with its receptors and ligands: the figure identifies the platelet (middle figure) together with its receptors, below: a2B for collagen, glycoprotein (GP) Ib/IX/V for von Willebrand factor (vWF), GP IIb/Illa for fibrinogen, PAR1 and PAR2 for thrombin, P2Y2-1 and P2Y2-2 for adenosine diphosphate (ADP), as well as receptors for epinephrine and thromboxane $A 2$

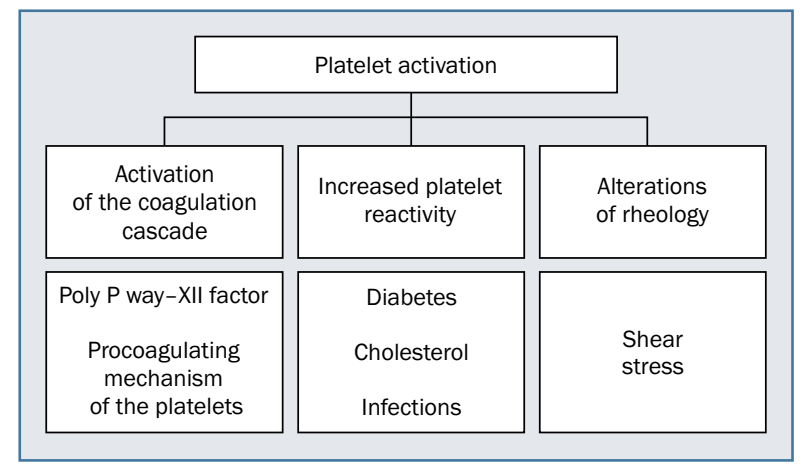

Figure 2. Mechanisms of platelet activation

\section{Conditions of conservation}

Under current guidelines, product stored as platelet concentrates (regardless of how it is obtained) must be kept under constant agitation at 60 oscillations per minute, as well as at a controlled temperature in the range $20-24^{\circ} \mathrm{C}$.

The estimated survival time is $3-7$ days; this is determined mainly by access to energy reserves in the form of adenosine triphosphate (ATP) obtained by platelets through glycolysis and oxidative phosphorylation. As a product of the above processes, lactic acid concentrations can rise to 3-5 times their basal value. However, the bicarbonate contained in the plasma on which the platelet concentrates are suspended exerts a neutralizing effect, thus maintaining the stability of the internal environment in said hemocomponent; the $\mathrm{pH}$ range under which platelets are considered viable is 6.4-7.4. 
There are reports in the literature that from a pH of 6.0 or less, platelets can change from their usual discoid shape to a spherical shape, which triggers a significant decrease in their survival. On the other hand, it is known that from day 7 there is the possibility that platelets preserved under the conventional method present bacterial contamination.

Another determining factor under current processing and conservation conditions is the type of storage to which the collected platelets are subjected, for which polyvinyl chloride (PVC) has been chosen as the preferred material for the manufacture of collecting bags of this blood component $[2,9]$.

\section{Problems associated with conventional preservation of platelets}

The first problem lies in the preservation conditions, taking into account that the useful life of each concentrate is related to strict compliance with them. That is why the requirement of maintaining a reduced thermal range, as well as the requirement of constant agitation, represents the first difficulty under the current conditions of conservation. To overcome this inconvenience, technological aid has been used, thus having shaking machines (marketed under different names) whose purpose is to maintain a constant 60-70 oscillations per minute. Some of these machines also function as incubators, keeping platelet concentrates in the range $20-24^{\circ} \mathrm{C}$, thus guaranteeing the fulfillment of these two vital conditions for the maintenance of this blood component.

The second problem is the risk of bacterial contamination related to the temperature at which platelet concentrates must be stored. To minimize this risk, basic measures must be used such as disinfection of the venipuncture area and the maintenance of closed circuits for collecting the platelets obtained. Likewise, the practice of diverting the first milliliters of the collected product to an intermediate bag has become common, after which the rest of the collected volume will be the one chosen for storage in the blood bank.

The third problem is the useful lifetime for each concentrate conserved under the method described above, which results in a frequent demand from donors in short periods of time while having higher production expenses and therefore higher costs. For this, the cryopreservation strategy arises, reviewed in the present work as an alternative for prolonging survival in collected platelets [1, 2].

\section{A strategy for platelet conservation: cryopreservation}

During the 1950s, the idea of prolonging the survival of stored platelets was conceived through the use of preservation techniques by freezing the concentrates with subsequent evaluation of both the platelet count and their functionality over time. For this, the study and testing of different substances to be used as a cryopreservative solution was also proposed, taking into account that the freezing process involved drastic changes in the cellular microenvironment, the main one evidenced in the intraand extracellular water content which goes from a liquid state to a solid state, generating water crystals that will cause mechanical damage to the membrane, thus triggering cell death.

Djerassi et al. implemented a first conservation model with positive results, taking $5 \%$ dimethylsulfoxide (DSMO) as a preservative solution and then cooling the platelet concentrates, decreasing by $1^{\circ} \mathrm{C}$ per min until freezing. Later, it was shown that patients undergoing cryopreserved platelet transfusion had adverse effects such as local vasospasm, nausea and vomiting; assuming that such effects were related to the DMSO contained in the cryoprotective solution, it was decided to implement post-thaw washing, thus eliminating the DMSO supernatant and thereby reducing the rate of adverse events. Even so, and despite the positive results that partially favored this new preservation procedure, it took about 20 years to carry out new research on the subject. Thus, in 1970 Valeri et al. developed a new method that consisted of using a mixture of platelet concentrates with 5-7\% DMSO as a preservative solution to then subject them to direct freezing at $-80^{\circ} \mathrm{C}$; this was extremely useful for use in field hospitals for the management of patients during military operations. Specifically, the results obtained from this work showed that at two years post-freezing, platelet recovery using 6\% DMSO as a cryopreservative was 74-94\%, depending on whether or not it was removed by washing with sodium chloride prior to freezing.

The first step of the Valeri technique consists of obtaining a concentrate by apheresis technique with $3.3 \times 10^{9}$ platelets in a total volume of $200 \mathrm{ml}$ solution to which $75 \mathrm{~mL}$ of solution of $25 \%$ DMSO in $0.9 \% \mathrm{NaCl}$, through sterile connector, resulting in a final DMSO concentration of 5-7\% for each platelet concentrate. Then the solution obtained should be centrifuged for 20 minutes at 3,600 revolutions per minute. Subsequently, the supernatant will be eliminated (with the objective of eliminating the DMSO content) obtaining a final product of $13-15 \mathrm{~mL}$ that contains the platelets that will be cryopreserved. This product should be placed in a freezer to be preserved at $-80^{\circ} \mathrm{C}$. The quality and characteristics of the product obtained will also depend on the correct fulfillment of this sequence of steps.

The process does not end only in the cryopreservation stage, taking into account that platelets must later be thawed and then reconstituted for use in the context of transfusion medicine. The thawing stage of the previously cryopreserved product should be carried out at $37^{\circ} \mathrm{C}$; the 
water bath method can be used during this step. Once thawed, platelets must be suspended in $0.9 \mathrm{NaCl}$ solution or plasma, which must be added using a sterile connector, after which the product to be used for transfusion will be obtained [10, 12].

The advantages offered by this method, based on the results described above, are: Ionger availability of stored platelets; lower risk of bacterial contamination; decrease in both the frequency and the time required for donors to supply the platelet demand of each institution; and optimization of resources and lower costs derived from less renewal of equipment and materials for the storage of platelet concentrates [10-13].

Cryopreserved concentrates have also been shown to be functionally competent after being transfused into healthy subjects. It is true that since it is a component prone to activation under minimal manipulation, it has been observed that in vivo cryopreserved platelets, once thawed, present morphological changes such as the loss of their discoid shape in addition to the elevation of P-selectin levels and exposure of phosphatidylserine and other microparticles in its outer membrane. However, there is no evidence of a greater risk of predisposition to thrombotic events compared to platelet concentrates stored conventionally.

The great difficulty encountered during these studies was that all the investigations had to be carried out with autologous platelets from people who participated, taking into account that by the 1970s there was still no availability of obtaining compatible human leukocyte antigen (HLA) units from other donors.

Reade et al. [14], in 2019, analyzed a population of 41 patients undergoing cardiovascular surgery, of whom 18 were transfused with platelet concentrates preserved in the conventional way, and 23 received cryopreserved platelets. The parameters were: blood loss during surgery and at 24 and 48 hours postoperatively, potential damages such as thrombosis were comparable to deep vein and myocardial infarction and the number of transfusion-related infections. With reference to bleeding, it was found that the mean bleeding in patients transfused with cryopreserved platelets was $925 \mathrm{~mL}$ during surgery and $140 \mathrm{~mL}$ obtained from drains during their admission to the intensive care unit (ICU) compared to $900 \mathrm{~mL}$ intraoperatively and $110 \mathrm{~mL}$ once admitted to the ICU in patients transfused with conventional platelets ( $p$ value 0.82 ). However, when compared at 24 and 48 hours after surgery, patients in the cryopreserved platelet group obtained lower blood output, reporting 715 and $980 \mathrm{~mL}$ versus 805 and 1,075 mL of the group transfused with non-cryopreserved platelets ( $p$ value 0.45 ). Finally, those transfused with previously frozen platelets had fewer cases requiring a second surgery due to bleeding 24-72 hours postoperatively, and also a lower report of critical bleeding [14].
A review by Slichter et al. [15] analyzed different studies which evaluated aspects of platelets such as their functionality and post-freezing in vitro survival, concluding that $28 \%$ of platelets are lost during cryopreservation, meaning that their survival is usually similar to that of fresh platelets. Likewise, platelets cryopreserved in DMSO have a hemostatic efficacy similar to that of platelets preserved under standard conditions [15].

Other studies carried out in patients with a transfusion requirement for other causes who received cryopreserved platelets have reported favorable results without evidence of adverse effects associated with the procedure $[13,16]$.

\section{Advantages of cryopreservation in a local context}

From the geographical point of view, Argentina has a vast territory $\left(2.78\right.$ million $\mathrm{km}^{2}$ ) divided into 23 provinces, with availability of hemotherapy and blood bank services limited to the Autonomous City of Buenos Aires (CABA), the provincial capitals and, with some exceptions, intermediate cities $[17,18]$. For this reason, platelet transport becomes difficult under the conditions of conservation described at the beginning of this article for health institutions located in remote regions of CABA or provincial capitals for which access to this therapeutic resource remains at present out of reach of residents far from urban centers.

All of the above has been magnified in the last 12 months by a situation of global magnitude, namely the pandemic generated by coronavirus disease 2019 (COVID-19) which has forced different countries to adopt measures such as mandatory quarantine which have caused a marked decrease in the number of donors, as well as the number of blood components available for institutional use [19].

That is why it is convenient and interesting to propose strategies that not only extend the duration, but also take greater advantage of the available resources that hemotherapy centers and blood banks currently have, especially in the case of platelets. Among them, cryopreservation could turn out to be a highly cost-effective method, since most of the materials are available, since they are part of the daily work and operation of centers that work with blood components.

In order to consider this strategy, we searched for reports in the literature that documented previous experiences in other countries on the implementation of techniques for the cryopreservation of platelets as well as their use in different clinical contexts. Cohn et al. [20] published a work in 2017 within the framework of the international forum for the cryopreservation of platelets. This covers a significant number of tests carried out by different countries in which parameters were: the methodology used during the preservation process; the evaluation in the time of 
the frozen product depending on its survival and functionality; and even in some cases the results obtained with respect to its use in human beings using as a comparator the transfusion of platelets preserved under conventional conditions [20].

As an example, the case of Australia will be cited, which implemented the strategy of platelet cryopreservation using the technique provided by Valeri, and limited the transfusion behavior to clinical trials carried out in members of the country's military, with encouraging results regarding their hemostatic function and time of platelet survival post-transfusion. The minimum requirements to consider the use of frozen platelets for transfusion in humans were: a count of at least $200 \times 10^{9}$ platelets for each post-thaw concentrate, as well as having shown that this count corresponded to a recovery of at least $40 \%$ compared to the count prior to freezing; and finally obtaining a pH of between 6.4 and 7.4. Similar experiences were documented in Belgium, Canada, the United States and France where, like Australia, its use was restricted to the military $[19,21]$.

On the other hand there is the Czech Republic where, in 2014, the transfusion of cryopreserved platelets in the civilian population was authorized. These platelets, like the previous examples, were preserved according to the Valeri method, prolonging their survival for up to four years. Among the requirements to consider them as suitable for use in humans were: obtaining $200-420 \times 10^{9}$ platelets post-thaw as well as documenting $\mathrm{pH}$ values greater than 6.4 for each unit. Among the indications under which cryopreserved platelets were transfused are: bleeding of traumatic origin, central nervous system hemorrhage and abdominal bleeding.

Following this example is Spain who adhered to the indication to transfuse cryopreserved platelets to patients undergoing liver transplantation [19, 22]. At present, there are no reports of research or experiences regarding the cryopreservation of platelets or the use of platelet concentrates preserved by freezing techniques in Argentina. From the Latin American perspective, the only mention is made in the work of Bohoněk [10] of a single pilot study carried out in a Brazilian hospital in which platelets were cryopreserved using the Valeri technique, obtaining a survival of two years in vitro. However, no other reports on similar studies were found, nor has there been any mention of trials that suggest the use of frozen platelets for subsequent transfusion in humans $[10,20]$.

The scarce information available, not only local but also regional, leads us to suggest the need for large-scale studies that allow not only experience within the field of cryopreservation but also facilitate its rapid implementation. This is a cost-effective alternative to solve the problem of the low availability of the mentioned blood component for the reasons discussed earlier.
Clearly, the benefits described, such as the reduced storage and conservation costs, as well as the less frequent requirement for donors, could be applicable to our context, also adding the possibility of even thinking about the transport of this component to distant populations of main cities. Finally, we hope to stimulate other investigations that allow us to deepen knowledge about new techniques to prolong useful survival and platelet functionality.

\section{References}

1. Salazar M. [Guidelines for the transfusion of blood and its components] [Article in Spanish]. Rev Panam Salud Publica. 2003; 13(2-3): 183-190, doi: 10.1590/s1020-49892003000200023, indexed in Pubmed: 12744795.

2. Verdugo $D^{\prime}$ hane M. Cost-benefit ratio of standard platelet transfusion versus apheresis platelets in the blood bank of Clinic Colsanitas SA; Master's thesis in health administration. Faculty of Administration, Universidad del Rosario, Bogota 2012: 28-40.

3. Izaguirre-Ávila R. The discovery of platelets. Rev Biomed. 1997(8): 197-208.

4. Izaguirre-Ávila R, de Micheli A. Evolution of the knowledge on the blood and its movement: part two. The knowledge about its composition. latrochemistry of the blood. Rev Invest Clín. 2005; 57(1): 85-97.

5. Gómez-Gómez B, Rodríguez-Weber FL, Díaz Greene EJ. Platelet physiology, platelet aggregometry and its clinical utility. Med Int Mex. 2018; 34(2): 244-263.

6. Jurk K, Kehrel BE. Platelets: physiology and biochemistry. Semin Thromb Hemost. 2005; 31(4): 381-392, doi: 10.1055/s-2005916671, indexed in Pubmed: 16149014.

7. Schultze M. Ein heizbarer Objecttisch und seine Verwendung bei Untersuchungen des Blutes. Arch Mikrosc Anat. 1865; 1: 1-42.

8. Gómez-Gómez B, Rodríguez-Weber FL, Díaz-Greene EJ. Platelet physiology, platelet aggregometry and its clinical utility. Int Med Mex. 2018; 34(2): $244-263$.

9. National Institute of Health. Hemovigilance manual. National Printing Office of Colombia, Bogota 2010: 11-77.

10. Bohoněk M. Cryopreservation of platelets: advances and current practice. Chapter 4. Cryopreservation Biotechnology in Biomedical and Biological Sciences, London 2018: 44-66.

11. Kelly K, Dumont L. Frozen platelets. Transfus Apher Sci. 2019; 58(1): 23-29, doi: 10.1016/j.transci.2018.12.013, indexed in Pubmed: 30612945.

12. Valeri $\mathrm{CR}$, Ragno $\mathrm{G}$, Khuri S. Freezing human platelets with 6 percent dimethyl sulfoxide with removal of the supernatant solution before freezing and storage at -80 degrees $C$ without postthaw processing. Transfusion. 2005; 45(12): 1890-1898, doi: 10.1111/j.15372995.2005.00647.x, indexed in Pubmed: 16371041.

13. Cohn CS, Williams S. Cryopreserved platelets: the thaw begins. Transfusion. 2019; 59(9): 2759-2762, doi: 10.1111/trf.15465.

14. Reade MC, et al. A randomized, controlled pilot clinical trial of cryopreserved platelets for perioperative surgical bleeding: the CLIP-I trial. Transfusion. 2019; 59(9): 27-94.

15. Slichter SJ, Jones M, Ransom J, et al. Review of in vivo studies of dimethyl sulfoxide cryopreserved platelets. Transfusion Medicine Reviews. 2014; 28(4): 212-225, doi: 10.1016/j. tmrv.2014.09.001. 
16. Slichter SJ, Dumont L, Cancelas J, et al. Safety and efficacy of cryopreserved platelets in bleeding patients with thrombocytopenia. Transfusion. 2018; 58(9): 2129-2138, doi: 10.1111/trf.14780.

17. Aparicio G. Polimodal Argentine geography. Pedagogical project with distance modality for completion of EGB3 and polimodal education EDITEP studies. Editorial of the National University of Cuyo, Ciudad de Mendoza 2005: 75-95.

18. Gutiérrez MG, de Tejada ES, Cruz JR. A study of sociocultural factors related to voluntary blood donation in the Americas. Pan Am J Public Health. 2003(2-3): 85-90.

19. Recommendations on the space for blood collection and the personnel involved. Argentine Ministry of Health 2020: 1-2.
20. Cohn CS, Dumont LJ, Lozano M, et al. Vox Sanguinis International Forum on platelet cryopreservation. Vox Sang. 2017; 112(7): e69-e85, doi: 10.1111/vox.12532, indexed in Pubmed: 28929500.

21. Reade MC, Marks DC, Johnson L, L, et al. Frozen platelets for rural Australia: the CLIP trial. Anaesth Intensive Care. 2013; 41(6): 804-805, indexed in Pubmed: 24180724.

22. Sanz C, Ghita G, Franquet C, et al. Red-blood-cell alloimmunization and female sex predict the presence of HLA antibodies in patients undergoing liver transplant. Vox Sang. 2010; 99(3): 261-266, doi: 10.1111/j.1423-0410.2010.01347.x, indexed in Pubmed: 20840340. 